Supporting Information for:

\title{
Exciton fission and fusion in bis(tetracene) molecules with different covalent linker structures
}

\author{
Astrid M. Müller ${ }^{(1)}$, Yuri S. Avlasevich ${ }^{(2)}$, Wolfgang W. Schoeller ${ }^{(1,3)}$, Klaus Müllen ${ }^{(2)}$, \\ and Christopher J. Bardeen ${ }^{(1) *}$ \\ ${ }^{(1)}$ Department of Chemistry \\ University of California \\ Riverside, CA 92521 \\ ${ }^{(2)}$ Max Planck Institute for Polymer Research \\ Ackermannweg 10 \\ D-55128 Mainz \\ Germany \\ ${ }^{(3)}$ Department of Chemistry \\ University of Bielefeld \\ D-33503 Bielefeld \\ Germany \\ *E-mail: christopher.bardeen@ucr.edu
}

Calculations. All quantum chemical calculations were performed with the Turbomole version 5-7 set of programs. ${ }^{1}$ Energy optimization of all structures was performed without any symmetry constraints at the B3LYP/SV(p) level of sophistication. ${ }^{2}$ For all calculations a higher grid $(\mathrm{m}=4)$ was employed, the SCF convergence was set to $10^{-7}$ au. The electronic excitations were throughout from the adiabatic approximation of time dependent density functional theory. ${ }^{3}$

The optimized geometries of all investigated molecular structures are given as follows in Cartesian coordinates. 


\section{Ground states $\left(\mathrm{S}_{\mathbf{0}}\right)$ :}

1,4-Bis(tetracen-5-yl)benzene (1)

C $\quad-3.3279803 \quad-1.0978328 \quad 7.1482892$

H $\quad-3.9456923 \quad-1.3070989 \quad 8.0275688$

C $\quad-1.9870670 \quad-0.8520990 \quad 7.2909948$

C $-1.1609115 \quad-0.5757290 \quad 6.1487569$

C $\quad-1.7748970 \quad-0.5624944 \quad 4.8332171$

$\begin{array}{llll}\text { C } & -3.1839236 & -0.8243492 & 4.7335667\end{array}$

C $-3.9354458-1.0834752 \quad 5.8504189$

$\mathrm{H} \quad-1.5212896 \quad-0.8621528 \quad 8.2820954$

C $\quad 0.2052613 \quad-0.3157650 \quad 6.2551181$

$\begin{array}{llll}\text { C } & -0.9881824 & -0.2973471 & 3.7096498\end{array}$

$\mathrm{H} \quad-3.6469693 \quad-0.8137990 \quad 3.7412755$

H $\quad-5.0080248 \quad-1.2820928 \quad 5.7578764$

C $\quad 1.0085338 \quad-0.0451799 \quad 5.1238516$

C $\quad 0.4009385 \quad-0.0410518 \quad 3.8018602$

$\begin{array}{llll}\text { C } & 2.3867249 & 0.2271771 & 5.2477209\end{array}$

$\begin{array}{llll}\text { C } & 1.2121076 & 0.2304220 & 2.6574322\end{array}$

C $3.1833081 \quad 0.4971112 \quad 4.1366254$

$\begin{array}{llll}\text { C } & 2.5883424 & 0.4964699 & 2.8091448\end{array}$

C $\quad 4.5871419 \quad 0.7722482 \quad 4.2711780$

$\begin{array}{llll}\text { C } & 3.4560760 & 0.7594925 & 1.6909196\end{array}$

$\begin{array}{llll}\text { C } & 5.3691970 & 1.0255822 & 3.1758709\end{array}$

C $\quad 4.7918665 \quad 1.0149919 \quad 1.8664863$

C $\quad 0.5907630 \quad 0.2309633 \quad 1.2946565$

C $\quad 0.2899887 \quad 1.4354261 \quad 0.6357266$

$\begin{array}{llll}\text { C } & 0.2886754 & -0.9738687 & 0.6362953\end{array}$

$\begin{array}{llll}\text { C } & -0.2909637 & 1.4353435 & -0.6360061\end{array}$

C $\quad-0.2903653 \quad-0.9739474 \quad-0.6359481$

C $\quad-0.5919709 \quad 0.2308033 \quad-1.2946711$

C $\quad-1.2127844 \quad 0.2301353 \quad-2.6576896$

$\begin{array}{llll}\text { C } & -2.5889457 & 0.4961229 & -2.8100425\end{array}$

C $-0.4010630 \quad-0.0413044 \quad-3.8017363$

C $-1.0080062-0.0453240 \quad-5.1240290$

C $\quad-3.1832863 \quad 0.4967775 \quad-4.1378014$

C $\quad-2.3861520 \quad 0.2269861 \quad-5.2485385$

$\begin{array}{llll}\text { C } & 0.9879970 & -0.2976786 & -3.7088552\end{array}$

C $\quad-0.2041632 \quad-0.3157803 \quad-6.2549254$

C $\quad 1.7752602 \quad-0.5627555 \quad-4.8320517$

C $\quad 1.1619500 \quad-0.5757880 \quad-6.1479056$

C $3.1842111 \quad-0.8247389 \quad-4.7316977$

$\begin{array}{llll}\text { C } & 1.9886866 & -0.8520496 & -7.2897507\end{array}$ 


$\begin{array}{rrrr}\text { C } & 3.9362965 & -1.0837876 & -5.8481877 \\ \mathrm{C} & 3.3295055 & -1.0979145 & -7.1463760 \\ \mathrm{C} & -3.4571850 & 0.7590972 & -1.6922038 \\ \mathrm{C} & -4.5870777 & 0.7718239 & -4.2729842 \\ \mathrm{C} & -4.7929105 & 1.0145103 & -1.8683721 \\ \mathrm{C} & -5.3696463 & 1.0250704 & -3.1780214 \\ \mathrm{H} & 0.6758144 & -0.3178492 & 7.2442221 \\ \mathrm{H} & 2.8395930 & 0.2259461 & 6.2448725 \\ \mathrm{H} & 5.0196055 & 0.7728131 & 5.2772552 \\ \mathrm{H} & 6.4374416 & 1.2331019 & 3.2945819 \\ \mathrm{H} & 5.4279118 & 1.2110710 & 0.9972468 \\ \mathrm{H} & 3.0321366 & 0.7511819 & 0.6843471 \\ \mathrm{H} & -1.4669229 & -0.2871850 & 2.7275824 \\ \mathrm{H} & 0.5143326 & 2.3868407 & 1.1283565 \\ \mathrm{H} & 0.5129831 & -1.9252951 & 1.1289363 \\ \mathrm{H} & -0.5150031 & 2.3866940 & -1.1288991 \\ \mathrm{H} & -0.5149169 & -1.9254357 & -1.1283585 \\ \mathrm{H} & -3.0336780 & 0.7508098 & -0.6854460 \\ \mathrm{H} & -5.4293645 & 1.2105508 & -0.9994232 \\ \mathrm{H} & -6.4378526 & 1.2325122 & -3.2972128 \\ \mathrm{H} & -5.0190879 & 0.7723858 & -5.2792563 \\ \mathrm{H} & -2.8385413 & 0.2258183 & -6.2459078 \\ \mathrm{H} & -0.6742150 & -0.3177427 & -7.2442682 \\ \mathrm{H} & 1.5234343 & -0.8619174 & -8.2811000 \\ \mathrm{H} & 3.9476592 & -1.3071165 & -8.0253602 \\ \mathrm{H} & 5.0088082 & -1.2825173 & -5.7551082 \\ \mathrm{H} & 3.6467361 & -0.8143476 & -3.7391621 \\ \mathrm{H} & 1.4662441 & -0.2876599 & -2.7265473\end{array}$

\begin{tabular}{|c|c|c|c|}
\hline $\mathrm{C}$ & 8.3442259 & 4.2089281 & -1.6103807 \\
\hline $\mathrm{H}$ & 9.0626834 & 4.9800633 & -1.9064810 \\
\hline $\mathrm{C}$ & 8.7823184 & 2.9611048 & -1.2501076 \\
\hline $\mathrm{C}$ & 7.8544729 & 1.9366740 & -0.8586554 \\
\hline $\mathrm{C}$ & 6.4364045 & 2.2483809 & -0.8525328 \\
\hline $\mathrm{C}$ & 6.0250379 & 3.5701536 & -1.2367254 \\
\hline $\mathrm{C}$ & 6.9449278 & 4.5181457 & -1.6032930 \\
\hline $\mathrm{H}$ & 9.8513426 & 2.7233825 & -1.2543792 \\
\hline $\mathrm{C}$ & 8.2632722 & 0.6574549 & -0.4817764 \\
\hline $\mathrm{C}$ & 5.5178193 & 1.2654860 & -0.4762061 \\
\hline $\mathrm{H}$ & 4.9555092 & 3.8051936 & -1.2308289 \\
\hline $\mathrm{H}$ & 6.6155786 & 5.5208873 & -1.8939354 \\
\hline $\mathrm{C}$ & 7.3411097 & -0.3429189 & -0.0983500 \\
\hline 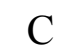 & 5.9184729 & -0.0384084 & -0.0980193 \\
\hline
\end{tabular}




$\begin{array}{lrrr}\mathrm{C} & 7.7683347 & -1.6301011 & 0.2888273 \\ \mathrm{C} & 4.9849907 & -1.0481700 & 0.2876634 \\ \mathrm{C} & 6.8633885 & -2.6193177 & 0.6692250 \\ \mathrm{C} & 5.4382516 & -2.3275571 & 0.6675554 \\ \mathrm{C} & 7.3055236 & -3.9289320 & 1.0615865 \\ \mathrm{C} & 4.5405784 & -3.3851558 & 1.0524308 \\ \mathrm{C} & 6.4115629 & -4.9003190 & 1.4261616 \\ \mathrm{C} & 5.0078562 & -4.6214592 & 1.4178597 \\ \mathrm{C} & 3.5195007 & -0.7386663 & 0.2873399 \\ \mathrm{C} & 2.8513031 & -0.3909025 & 1.4738493 \\ \mathrm{C} & 2.7678247 & -0.7854631 & -0.8993974 \\ \mathrm{C} & 1.4847872 & -0.1030935 & 1.4741133 \\ \mathrm{C} & 1.4010202 & -0.4989708 & -0.8989674 \\ \mathrm{C} & 0.7278223 & -0.1511956 & 0.2878894 \\ \mathrm{C} & -0.7283181 & 0.1532092 & 0.2889424 \\ \mathrm{C} & -1.5537354 & -0.2305439 & 1.3632489 \\ \mathrm{C} & -1.3331820 & 0.8359216 & -0.7836142 \\ \mathrm{C} & -2.9206307 & 0.0549503 & 1.3646953 \\ \mathrm{C} & -2.6999842 & 1.1228676 & -0.7814172 \\ \mathrm{C} & -3.5203586 & 0.7386595 & 0.2931222 \\ \mathrm{C} & -4.9862533 & 1.0459110 & 0.2964042 \\ \mathrm{C} & -5.4412626 & 2.3220505 & 0.6847990 \\ \mathrm{C} & -5.9183555 & 0.0373219 & -0.0957850 \\ \mathrm{C} & -6.8664532 & 2.6133515 & 0.6840006 \\ \mathrm{C} & -7.3418828 & 0.3376179 & -0.0870017 \\ \mathrm{C} & -7.7705111 & 1.6233166 & 0.3036048 \\ \mathrm{H} & 4.4544246 & 1.5167507 & -0.4736174 \\ \mathrm{C} & -5.5144663 & -1.2556605 & -0.5066284 \\ \mathrm{C} & -8.2629787 & -0.6625531 & -0.4735162 \\ \mathrm{C} & -6.4318588 & -2.2375462 & -0.8884996 \\ \mathrm{C} & -7.8517951 & -1.9350151 & -0.8700704 \\ \mathrm{C} & -6.0172705 & -3.5487988 & -1.3040104 \\ \mathrm{C} & -8.7785259 & -2.9590956 & -1.2650510 \\ \mathrm{C} & -6.9361805 & -4.4966660 & -1.6733777 \\ \mathrm{C} & -8.3375263 & -4.1974687 & -1.6532689 \\ \mathrm{C} & -4.5464318 & 3.3702824 & 1.1005317 \\ \mathrm{C} & -7.3099487 & 3.9208914 & 1.0817574 \\ \mathrm{C} & -5.0151665 & 4.6036483 & 1.4738958 \\ \mathrm{C} & -6.4178518 & 4.8875356 & 1.4631992 \\ \mathrm{H} & 9.3314840 & 0.4151851 & -0.4792541 \\ \mathrm{H} & -0.3363482 & 2.4104088\end{array}$




$\begin{array}{rrrr}H & 3.2614547 & -1.0627818 & -1.8361466 \\ H & 1.0025345 & 0.1890481 & 2.4117632 \\ H & 0.8414152 & -0.5730853 & -1.8362746 \\ H & -1.1245981 & -0.7834237 & 2.2042615 \\ H & -0.7203398 & 1.1709206 & -1.6258766 \\ H & -3.5379710 & -0.2625359 & 2.2110315 \\ H & -3.1403997 & 1.6618169 & -1.6261907 \\ H & -4.9461636 & -3.7761693 & -1.3189321 \\ H & -6.6044366 & -5.4916211 & -1.9871273 \\ H & -9.0551653 & -4.9684423 & -1.9517662 \\ H & -9.8490329 & -2.7286323 & -1.2498127 \\ H & -4.4492398 & -1.4979949 & -0.5289467 \\ H & -9.3320585 & -0.4244802 & -0.4608982 \\ H & -8.8427598 & 1.8462335 & 0.3067411 \\ H & -8.3855101 & 4.1267179 & 1.0737329 \\ H & -6.7713567 & 5.8789061 & 1.7640973 \\ H & -4.3116992 & 5.3820542 & 1.7865782 \\ H & -3.4731187 & 3.1680490 & 1.1176288\end{array}$

1,3-Bis(tetracen-5-yl)benzene (3)

$\begin{array}{cccc}\mathrm{C} & -4.8379233 & 0.3065714 & 4.8744555 \\ \mathrm{H} & -5.7625941 & 0.5095553 & 5.4242151 \\ \mathrm{C} & -3.6353392 & 0.7477540 & 5.3622463 \\ \mathrm{C} & -2.4117257 & 0.4911914 & 4.6547957 \\ \mathrm{C} & -2.4720576 & -0.2485779 & 3.4069398 \\ \mathrm{C} & -3.7552067 & -0.6899703 & 2.9353627 \\ \mathrm{C} & -4.8988259 & -0.4229790 & 3.6425034 \\ \mathrm{H} & -3.5870898 & 1.3057829 & 6.3033094 \\ \mathrm{C} & -1.1705371 & 0.9264372 & 5.1191753 \\ \mathrm{C} & -1.2930778 & -0.5089387 & 2.7045255 \\ \mathrm{H} & -3.8003157 & -1.2476281 & 1.9938028 \\ \mathrm{H} & -5.8691906 & -0.7661786 & 3.2696596 \\ \mathrm{C} & 0.0273729 & 0.6682147 & 4.4146151 \\ \mathrm{C} & -0.0268987 & -0.0762367 & 3.1659306 \\ \mathrm{C} & 1.2759543 & 1.1173671 & 4.8928297 \\ \mathrm{C} & 1.1818079 & -0.3394295 & 2.4529844 \\ \mathrm{C} & 2.4593813 & 0.8653385 & 4.2012972 \\ \mathrm{C} & 2.4164744 & 0.1192418 & 2.9533017 \\ \mathrm{C} & 3.7284589 & 1.3245422 & 4.6942785 \\ \mathrm{C} & 3.6643852 & -0.1249307 & 2.2788099 \\ \mathrm{C} & 4.8890176 & 1.0694128 & 4.0133178 \\ \mathrm{C} & 4.8530212 & 0.3318095 & 2.7873522 \\ \mathrm{C} & 1.1280166 & -1.1100515 & 1.1684142 \\ \mathrm{C} & 1.0675986 & -0.4410466 & -0.0660598\end{array}$




\begin{tabular}{|c|c|c|c|}
\hline & 558 & 8 & \\
\hline & 1.0148280 & & \\
\hline & & & \\
\hline & & & \\
\hline & & & \\
\hline & 79 & & \\
\hline & & & \\
\hline & & & \\
\hline & & & \\
\hline & 4921 & & \\
\hline & & & \\
\hline & & & \\
\hline & & & \\
\hline & & & \\
\hline & & & \\
\hline & & & \\
\hline & & & \\
\hline & & & \\
\hline & & & \\
\hline & & & \\
\hline & & & \\
\hline & & & \\
\hline & & & \\
\hline & & & \\
\hline & & & \\
\hline & & & \\
\hline & & & \\
\hline $\mathrm{H}$ & & & \\
\hline & & & \\
\hline & & & \\
\hline & & & \\
\hline & & & \\
\hline & & & \\
\hline & & & \\
\hline & & & \\
\hline $\mathrm{H}$ & -4.1 & & \\
\hline & & & \\
\hline & & & \\
\hline & & & \\
\hline & & & 62 \\
\hline $\mathrm{H}$ & 27 & & 806 \\
\hline & & & \\
\hline & & & \\
\hline & & & \\
\hline
\end{tabular}




\section{Excited singlet states $\left(\mathbf{S}_{1}\right)$ :}

$\begin{array}{lrrr}\text { 1,4-Bis(tetracen-5-yl)benzene } & (\mathbf{1}) \\ & & & \\ \text { C } & -3.2981884 & -2.5327831 & 6.6243444 \\ \text { H } & -3.8806450 & -3.0212552 & 7.4116704 \\ \text { C } & -2.0669953 & -1.9844279 & 6.9158468 \\ \text { C } & -1.2931996 & -1.3435125 & 5.9002366 \\ \text { C } & -1.8225732 & -1.2818584 & 4.5554644 \\ \text { C } & -3.1033286 & -1.8549133 & 4.2962831 \\ \text { C } & -3.8225026 & -2.4663505 & 5.3030892 \\ \text { H } & -1.6651587 & -2.0315598 & 7.9331425 \\ \text { C } & -0.0467255 & -0.7541756 & 6.1565452 \\ \text { C } & -1.0674874 & -0.6592075 & 3.5436796 \\ \text { H } & -3.5059449 & -1.8040704 & 3.2796146 \\ \text { H } & -4.8023390 & -2.9037337 & 5.0884567 \\ \text { C } & 0.7101160 & -0.1239667 & 5.1450525 \\ \text { C } & 0.2049212 & -0.0993823 & 3.7798814 \\ \text { C } & 1.9343085 & 0.5051512 & 5.4375805 \\ \text { C } & 0.9804500 & 0.5257502 & 2.7463702 \\ \text { C } & 2.6944469 & 1.1413883 & 4.4455933 \\ \text { C } & 2.2272193 & 1.1409724 & 3.0730471 \\ \text { C } & 3.9381582 & 1.7683603 & 4.7615979 \\ \text { C } & 3.0805745 & 1.7253353 & 2.0874293 \\ \text { C } & 4.7118036 & 2.3527076 & 3.7814053 \\ \text { C } & 4.2808705 & 2.3190859 & 2.4300065 \\ \text { C } & 0.4866383 & 0.5278697 & 1.3478666 \\ \text { C } & 0.2305433 & 1.7349429 & 0.6638224 \\ \text { C } & 0.2509358 & -0.6776769 & 0.6531894 \\ \text { C } & -0.2542511 & 1.7344341 & -0.6426347 \\ \text { C } & -0.2027742 & -0.6740759 & -0.6640795 \\ \text { C } & -0.4772380 & 0.5309977 & -1.3478198 \\ \text { C } & -0.9755515 & 0.5310702 & -2.7435086 \\ \text { C } & -2.2371049 & 1.1467215 & -3.0493647 \\ \text { C } & -0.1941149 & -0.0899670 & -3.7754712 \\ \text { C } & -0.7119900 & -0.1132331 & -5.1392092 \\ \text { C } & -2.7123032 & 1.1428783 & -4.4181202 \\ \text { C } & -1.9428329 & 0.5098690 & -5.4143691 \\ \text { C } & 1.0839286 & -0.6429006 & -3.5594125 \\ \text { C } & 0.0411043 & -0.7384125 & -6.1563466 \\ \text { C } & 1.8409368 & -1.2666743 & -4.5808633 \\ \text { C } & 1.2990188 & -1.3275106 & -5.9196605 \\ \text { C } & 3.1204690 & -1.8351296 & -4.3392073 \\ \text { C } & 2.0633236 & -1.9617970 & -6.9394442 \\ \text { C } & 3.8408228 & -2.4470239 & -5.3574403 \\ \text { C } & 3.3073632 & -2.5118392 & -6.6656973\end{array}$




$\begin{array}{rrrr}\mathrm{C} & -3.0899135 & 1.7228334 & -2.0666446 \\ \mathrm{C} & -3.9587995 & 1.7564199 & -4.7252181 \\ \mathrm{C} & -4.3056053 & 2.3123832 & -2.4003992 \\ \mathrm{C} & -4.7426904 & 2.3401369 & -3.7407129 \\ \mathrm{H} & 0.3524632 & -0.7723532 & 7.1760689 \\ \mathrm{H} & 2.3027203 & 0.4971024 & 6.4685207 \\ \mathrm{H} & 4.2705770 & 1.7686407 & 5.8046706 \\ \mathrm{H} & 5.6632703 & 2.8277989 & 4.0393545 \\ \mathrm{H} & 4.9095606 & 2.7577388 & 1.6491099 \\ \mathrm{H} & 2.7818998 & 1.6903345 & 1.0386444 \\ \mathrm{H} & -1.4980809 & -0.6033858 & 2.5422555 \\ \mathrm{H} & 0.3965320 & 2.6884038 & 1.1749800 \\ \mathrm{H} & 0.4477870 & -1.6322423 & 1.1510693 \\ \mathrm{H} & -0.4500890 & 2.6875566 & -1.1422107 \\ \mathrm{H} & -0.3658876 & -1.6253972 & -1.1783059 \\ \mathrm{H} & -2.7924793 & 1.6936327 & -1.0165823 \\ \mathrm{H} & -4.9277714 & 2.7493024 & -1.6124573 \\ \mathrm{H} & -5.6984620 & 2.8065513 & -4.0000532 \\ \mathrm{H} & -4.2956122 & 1.7516934 & -5.7674446 \\ \mathrm{H} & -2.3179818 & 0.5018913 & -6.4435073 \\ \mathrm{H} & -0.3685256 & -0.7551158 & -7.1722520 \\ \mathrm{H} & 1.6513754 & -2.0065918 & -7.9534248 \\ \mathrm{H} & 3.8797792 & -2.9953968 & -7.4639716 \\ \mathrm{H} & 4.8243165 & -2.8800797 & -5.1479746 \\ \mathrm{H} & 3.5343865 & -1.7852633 & -3.3261017 \\ \mathrm{H} & 1.5330640 & -0.5871586 & -2.5655419\end{array}$

$\begin{array}{cccc}\text { 4,4'-Bis(tetracen-5-yl)biphenylene (2) } \\ \text { C } & 8.3143197 & 1.7984761 & 4.2064750 \\ \text { H } & 9.0360295 & 2.1329380 & 4.9589894 \\ \text { C } & 8.7563504 & 1.4115439 & 2.9365180 \\ \text { C } & 7.8437003 & 0.9753504 & 1.9455196 \\ \text { C } & 6.4342091 & 0.9350021 & 2.2645781 \\ \text { C } & 6.0190313 & 1.3291989 & 3.5582976 \\ \text { C } & 6.9443929 & 1.7567027 & 4.5183603 \\ \text { H } & 9.8248136 & 1.4406369 & 2.6949042 \\ \text { C } & 8.2487230 & 0.5624024 & 0.6517118 \\ \text { C } & 5.5173326 & 0.4969023 & 1.2689913 \\ \text { H } & 4.9514311 & 1.2964064 & 3.8031600 \\ \text { H } & 6.6014707 & 2.0582193 & 5.5135378 \\ \text { C } & 7.3375784 & 0.1277746 & -0.3338702 \\ \text { C } & 5.9146290 & 0.1095176 & -0.0278195 \\ \text { C } & 7.7704569 & -0.3023952 & -1.6014240 \\ \text { C } & 4.9915505 & -0.3215452 & -1.0282913\end{array}$




\begin{tabular}{|c|c|c|c|}
\hline $\mathrm{C}$ & 6.8729441 & -0.7449590 & -2.6040002 \\
\hline $\mathrm{C}$ & 5.4520946 & -0.7488411 & -2.3237073 \\
\hline $\mathrm{C}$ & 7.3290345 & -1.1660298 & -3.8768831 \\
\hline $\mathrm{C}$ & 4.5766475 & -1.1518428 & -3.3624790 \\
\hline $\mathrm{C}$ & 6.4368811 & -1.5769217 & -4.8720076 \\
\hline $\mathrm{C}$ & 5.0579891 & -1.5636737 & -4.6131053 \\
\hline $\mathrm{C}$ & 3.5314027 & -0.3249196 & -0.7293167 \\
\hline $\mathrm{C}$ & 2.8151003 & -1.5305802 & -0.5947029 \\
\hline $\mathrm{C}$ & 2.8170964 & 0.8791504 & -0.5709522 \\
\hline $\mathrm{C}$ & 1.4480933 & -1.5315616 & -0.3124838 \\
\hline $\mathrm{C}$ & 1.4488746 & 0.8772531 & -0.2942942 \\
\hline $\mathrm{C}$ & 0.7310267 & -0.3279376 & -0.1565479 \\
\hline $\mathrm{C}$ & -0.7255487 & -0.3300916 & 0.1417371 \\
\hline $\mathrm{C}$ & -1.5840327 & -1.3017934 & -0.4103192 \\
\hline $\mathrm{C}$ & -1.3005197 & 0.6387745 & 0.9879912 \\
\hline $\mathrm{C}$ & -2.9525727 & -1.3023723 & -0.1318342 \\
\hline $\mathrm{C}$ & -2.6683373 & 0.6344405 & 1.2709951 \\
\hline $\mathrm{C}$ & -3.5228485 & -0.3356773 & 0.7164811 \\
\hline $\mathrm{C}$ & -4.9893098 & -0.3394165 & 1.0175648 \\
\hline $\mathrm{C}$ & -5.4528093 & -0.78 & 2.2816940 \\
\hline $\mathrm{C}$ & -5.9173799 & 0.1055195 & 0.0275481 \\
\hline $\mathrm{C}$ & -6.8795990 & -0.7806371 & 2.5688478 \\
\hline $\mathrm{C}$ & -7.3443302 & 0.0908652 & 0.3275750 \\
\hline $\mathrm{C}$ & -7.7765638 & -0.3515225 & 1.5946045 \\
\hline $\mathrm{C}$ & -5.5134380 & 0.5862539 & -1.2414857 \\
\hline $\mathrm{C}$ & -8.2626487 & 0.5305998 & -0.6533137 \\
\hline $\mathrm{C}$ & -6.4293580 & 1.0211716 & -2.2035532 \\
\hline $\mathrm{C}$ & -7.8508576 & 0.9888387 & -1.9034208 \\
\hline $\mathrm{C}$ & -6.0133566 & 1.5079840 & -3.4881893 \\
\hline $\mathrm{C}$ & -8.7741924 & 1.4404668 & -2.9053990 \\
\hline $\mathrm{C}$ & -6.9284370 & 1.9294050 & -4.4136534 \\
\hline $\mathrm{C}$ & -8.3291568 & 1.8950004 & -4.1195170 \\
\hline $\mathrm{C}$ & -4.5687183 & -1.2664461 & 3.3072702 \\
\hline $\mathrm{C}$ & -7.3314855 & -1.2334741 & 3.8539506 \\
\hline $\mathrm{C}$ & -5.0466649 & -1.6928834 & 4.5212869 \\
\hline $\mathrm{C}$ & -6.4478949 & -1.6729136 & 4.8029745 \\
\hline $\mathrm{H}$ & 9.3169558 & 0.5774180 & 0.4065582 \\
\hline $\mathrm{H}$ & 8.8439276 & -0.2956675 & -1.8220851 \\
\hline $\mathrm{H}$ & 8.4068953 & -1.1605494 & -4.0740985 \\
\hline $\mathrm{H}$ & 6.8118186 & -1.8989435 & -5.8490029 \\
\hline $\mathrm{H}$ & 4.3529105 & -1.8727899 & -5.3917220 \\
\hline $\mathrm{H}$ & 3.4981012 & -1.1340666 & -3.1875144 \\
\hline $\mathrm{H}$ & 4.4575506 & 0.4532418 & 1.5339330 \\
\hline $\mathrm{H}$ & 3.3456825 & -2.4827569 & -0.6979168 \\
\hline $\mathrm{H}$ & 3.3440022 & 1.8321951 & -0.6842165 \\
\hline $\mathrm{H}$ & 0.9331180 & -2.4884064 & -0.179937 \\
\hline
\end{tabular}


$\begin{array}{llll}\mathrm{H} & 0.9214793 & 1.8330635 & -0.2125264\end{array}$

$\begin{array}{cccc}\text { 1,3-Bis(tetracen-5-yl)benzene } & (\mathbf{3}) \\ \text { C } & -4.0147697 & -0.8292756 & 4.8917118 \\ \text { H } & -4.9200546 & -0.7780600 & 5.5062602 \\ \text { C } & -2.9047493 & -0.0651024 & 5.2223976 \\ \text { C } & -1.7063828 & -0.1126668 & 4.4525370 \\ \text { C } & -1.6657831 & -0.9766937 & 3.2894129 \\ \text { C } & -2.8192361 & -1.7591320 & 2.9921097 \\ \text { C } & -3.9703642 & -1.6885819 & 3.7684097 \\ \text { H } & -2.9316371 & 0.5904243 & 6.1005154 \\ \text { C } & -0.5511576 & 0.6274966 & 4.7792687 \\ \text { C } & -0.4894009 & -1.0108922 & 2.5035413 \\ \text { H } & -2.7772635 & -2.4470703 & 2.1382756 \\ \text { H } & -4.8370569 & -2.3124085 & 3.5225785 \\ \text { C } & 0.6337416 & 0.5838496 & 4.0100480 \\ \text { C } & 0.6663399 & -0.2509149 & 2.8093882 \\ \text { C } & 1.7865307 & 1.3157833 & 4.3581387 \\ \text { C } & 1.8488230 & -0.2519222 & 2.0156028 \\ \text { C } & 2.9704914 & 1.2869340 & 3.5930011 \\ \text { C } & 3.0082793 & 0.4890855 & 2.3825860 \\ \text { C } & 4.1307039 & 2.0267541 & 3.9628691 \\ \text { C } & 4.2120718 & 0.5009017 & 1.6179840 \\ \text { C } & 5.2843890 & 2.0066025 & 3.1959047 \\ \text { C } & 5.3220469 & 1.2357147 & 2.0113784 \\ \text { C } & 1.8194811 & -1.0068434 & 0.7242561 \\ \text { C } & 1.2667062 & -0.4008239 & -0.4160816 \\ \text { C } & 2.2595787 & -2.3360084 & 0.6040438 \\ \text { C } & 1.1204741 & -1.0892866 & -1.6336477 \\ \text { C } & 2.1364989 & -3.0351889 & -0.6029751 \\ \text { C } & 1.5667746 & -2.4230192 & -1.7208735 \\ \text { C } & 0.4636624 & -0.4306582 & -2.7945758 \\ \text { C } & 1.1641109 & -0.2627805 & -4.0322599 \\ \text { C } & -0.8815154 & 0.0446648 & -2.6872688 \\ \text { C } & 0.5001560 & 0.3382923 & -5.1679237 \\ \text { C } & -1.5219901 & 0.6836836 & -3.8257969 \\ \text { C } & -0.8228066 & 0.7981610 & -5.0379300 \\ \text { C } & 2.5304231 & -0.6351789 & -4.1828773 \\ \text { C } & 1.2052105 & 0.4849533 & -6.3957975 \\ \text { C } & 3.1900978 & -0.4708978 & -5.3893416 \\ \text { C } & 2.5222510 & 0.0817714 & -6.5080449 \\ \text { C } & -1.6370884 & -0.0990048 & -1.5038572 \\ \text { C } & -2.8416981 & 1.1685239 & -3.7043738 \\ \text { C } & -2.9503862 & 0.4014021 & -1.3799727\end{array}$




$\begin{array}{rrrr}\text { C } & -3.5709986 & 1.0560002 & -2.5073712 \\ \mathrm{C} & -3.6734160 & 0.2780170 & -0.1616936 \\ \mathrm{C} & -4.8958090 & 1.5566033 & -2.3697269 \\ \mathrm{C} & -4.9602598 & 0.7813649 & -0.0624815 \\ \mathrm{C} & -5.5721556 & 1.4207279 & -1.1696863 \\ \mathrm{H} & -0.5681044 & 1.2647105 & 5.6707139 \\ \mathrm{H} & 1.7603075 & 1.9339977 & 5.2625828 \\ \mathrm{H} & 4.0907062 & 2.6228664 & 4.8817377 \\ \mathrm{H} & 6.1626249 & 2.5836213 & 3.5044955 \\ \mathrm{H} & 6.2319152 & 1.2152845 & 1.4014055 \\ \mathrm{H} & 4.2638056 & -0.0922788 & 0.7000822 \\ \mathrm{H} & -0.4704897 & -1.6950507 & 1.6495823 \\ \mathrm{H} & 4.2408344 & -0.7618679 & -5.4745773 \\ \mathrm{H} & 3.0654817 & -1.0437838 & -3.3252723 \\ \mathrm{H} & 3.0549740 & 0.2035334 & -7.4555759 \\ \mathrm{H} & 0.6877170 & 0.9332604 & -7.2492954 \\ \mathrm{H} & -1.3132086 & 1.2648111 & -5.8976690 \\ \mathrm{H} & -3.3078502 & 1.6493689 & -4.5701633 \\ \mathrm{H} & -5.3704106 & 2.0516601 & -3.2223297 \\ \mathrm{H} & -6.5893864 & 1.8112068 & -1.0720368 \\ \mathrm{H} & -5.5032170 & 0.6845101 & 0.8811494 \\ \mathrm{H} & -3.2054825 & -0.2074315 & 0.6996613 \\ \mathrm{H} & -1.2010539 & -0.6065168 & -0.6432887 \\ \mathrm{H} & 1.4419461 & -2.9862627 & -2.6509187 \\ \mathrm{H} & 2.4716191 & -4.0753770 & -0.6688370 \\ \mathrm{H} & 2.6891493 & -2.8296842 & 1.4805905 \\ \mathrm{H} & 0.9409481 & 0.6402737 & -0.3359162\end{array}$

\section{Excited triplet states $\left(\mathrm{T}_{1}\right)$ :}

1,4-Bis(tetracen-5-yl)benzene (1)

$\begin{array}{cccc}\mathrm{C} & -3.3251422 & -1.2973680 & 7.0947892 \\ \mathrm{H} & -3.9427102 & -1.5422328 & 7.9649251 \\ \mathrm{C} & -1.9954772 & -1.0050001 & 7.2539855 \\ \mathrm{C} & -1.1696978 & -0.6812669 & 6.1239705 \\ \mathrm{C} & -1.7719004 & -0.6705687 & 4.8029184 \\ \mathrm{C} & -3.1692998 & -0.9826867 & 4.6858135 \\ \mathrm{C} & -3.9209117 & -1.2858332 & 5.7914710 \\ \mathrm{H} & -1.5386678 & -1.0129890 & 8.2492702 \\ \mathrm{C} & 0.1851213 & -0.3735253 & 6.2474099 \\ \mathrm{C} & -0.9854473 & -0.3591304 & 3.6911128 \\ \mathrm{H} & -3.6233456 & -0.9742631 & 3.6893550 \\ \mathrm{H} & -4.9845773 & -1.5221820 & 5.6856878\end{array}$




\begin{tabular}{|c|c|c|c|}
\hline $\mathrm{C}$ & 0.9879943 & -0.0556702 & 5.1282150 \\
\hline $\mathrm{C}$ & 0.3920038 & -0.0520622 & 3.8009933 \\
\hline $\mathrm{C}$ & 2.3544703 & 0.2639076 & 5.2687943 \\
\hline $\mathrm{C}$ & 1.2021759 & 0.2685881 & 2.6689294 \\
\hline $\mathrm{C}$ & 3.1499654 & 0.5812778 & 4.1694408 \\
\hline $\mathrm{C}$ & 2.5660980 & 0.5825360 & 2.8370752 \\
\hline $\mathrm{C}$ & 4.5418070 & 0.9045470 & 4.3204662 \\
\hline $\mathrm{C}$ & 3.4314441 & 0.8989464 & 1.7309672 \\
\hline $\mathrm{C}$ & 5.3223026 & 1.2062461 & 3.2363086 \\
\hline $\mathrm{C}$ & 4.7555792 & 1.1997733 & 1.9222108 \\
\hline $\mathrm{C}$ & 0.5923498 & 0.2697277 & 1.3009171 \\
\hline $\mathrm{C}$ & 0.2201783 & 1.4717222 & 0.6743294 \\
\hline $\mathrm{C}$ & 0.3719657 & -0.9316810 & 0.6054168 \\
\hline $\mathrm{C}$ & -0.3530910 & 1.4724716 & -0.6003024 \\
\hline $\mathrm{C}$ & -0.1957327 & -0.9310243 & -0.6717301 \\
\hline $\mathrm{C}$ & -0.5706580 & 0.2710703 & -1.2992918 \\
\hline $\mathrm{C}$ & -1.1816800 & 0.2707901 & -2.6611684 \\
\hline $\mathrm{C}$ & -2.5914592 & 0.5910645 & -2.80 \\
\hline $\mathrm{C}$ & -0.3829496 & -0.0448053 & -3.7979457 \\
\hline $\mathrm{C}$ & -0.9989835 & -0.0540066 & -5.1264320 \\
\hline $\mathrm{C}$ & -3.1780368 & 0.5909562 & -4.1212322 \\
\hline $\mathrm{C}$ & -2.3610566 & 0.2648709 & -5.2491889 \\
\hline $\mathrm{C}$ & 0.9921042 & -0.3289520 & -3.7115855 \\
\hline $\mathrm{C}$ & -0.2100097 & -0.3706029 & -6.2472401 \\
\hline $\mathrm{C}$ & 1.7978616 & -0.6517464 & -4.8540568 \\
\hline $\mathrm{C}$ & 1.1843746 & -0.6777956 & -6.1514368 \\
\hline $\mathrm{C}$ & 3.1718343 & -0.9429719 & -4.7442276 \\
\hline $\mathrm{C}$ & 1.9730029 & -0.9960153 & -7.2753319 \\
\hline $\mathrm{C}$ & 3.9352938 & -1.2563651 & -5.8741179 \\
\hline $\mathrm{C}$ & 3.3351946 & -1.2833174 & -7.1403093 \\
\hline $\mathrm{C}$ & -3.4259034 & 0.8828434 & -1.7096587 \\
\hline $\mathrm{C}$ & -4.5449626 & 0.8989315 & -4.2686101 \\
\hline $\mathrm{C}$ & -4.7830226 & 1.1859756 & -1.8809837 \\
\hline $\mathrm{C}$ & -5.3450727 & 1.1981766 & -3.1612784 \\
\hline $\mathrm{H}$ & 0.6465554 & -0.3744163 & 7.2408070 \\
\hline $\mathrm{H}$ & 2.7988398 & 0.2620645 & 6.2697684 \\
\hline $\mathrm{H}$ & 4.9664298 & 0.9024102 & 5.3298789 \\
\hline $\mathrm{H}$ & 6.3813619 & 1.4500952 & 3.3675618 \\
\hline $\mathrm{H}$ & 5.3903845 & 1.4360964 & 1.0621323 \\
\hline $\mathrm{H}$ & 3.0151502 & 0.8949772 & 0.7211229 \\
\hline $\mathrm{H}$ & -1.4548222 & -0.3522174 & 2.7044713 \\
\hline $\mathrm{H}$ & 0.3827630 & 2.4206858 & 1.1951681 \\
\hline $\mathrm{H}$ & 0.6514654 & -1.8812765 & 1.0727525 \\
\hline $\mathrm{H}$ & -0.6329007 & 2.4225401 & -1.0662570 \\
\hline $\mathrm{H}$ & -0.3568412 & -1.8800999 & -1.1924857 \\
\hline $\mathrm{H}$ & -3.0066616 & 0.8697304 & -0.7012244 \\
\hline
\end{tabular}




$\begin{array}{rrrr}\mathrm{H} & -5.4007400 & 1.4102759 & -1.0056858 \\ \mathrm{H} & -6.4048047 & 1.4348893 & -3.2987996 \\ \mathrm{H} & -4.9792869 & 0.8974561 & -5.2739210 \\ \mathrm{H} & -2.8185715 & 0.2638661 & -6.2439301 \\ \mathrm{H} & -0.6810818 & -0.3784977 & -7.2358233 \\ \mathrm{H} & 1.5043970 & -1.0151315 & -8.2650320 \\ \mathrm{H} & 3.9310984 & -1.5281692 & -8.0252153 \\ \mathrm{H} & 5.0013677 & -1.4799870 & -5.7668263 \\ \mathrm{H} & 3.6412248 & -0.9222427 & -3.7549839 \\ \mathrm{H} & 1.4826693 & -0.3021873 & -2.7360039\end{array}$

4,4'-Bis(tetracen-5-yl)biphenylene (2)

$\begin{array}{lrrr}\mathrm{C} & 8.3809584 & 4.2639986 & -1.3239407 \\ \mathrm{H} & 9.1050045 & 5.0479041 & -1.5684009 \\ \mathrm{C} & 8.8111394 & 2.9960219 & -1.0306738 \\ \mathrm{C} & 7.8758047 & 1.9546743 & -0.7076836 \\ \mathrm{C} & 6.4588278 & 2.2709508 & -0.6985424 \\ \mathrm{C} & 6.0558979 & 3.6141523 & -1.0106349 \\ \mathrm{C} & 6.9827014 & 4.5778147 & -1.3134208 \\ \mathrm{H} & 9.8793493 & 2.7547627 & -1.0374041 \\ \mathrm{C} & 8.2761899 & 0.6547498 & -0.3988885 \\ \mathrm{C} & 5.5329735 & 1.2717322 & -0.3887250 \\ \mathrm{H} & 4.9871722 & 3.8527477 & -1.0021269 \\ \mathrm{H} & 6.6597444 & 5.5967415 & -1.5498876 \\ \mathrm{C} & 7.3465783 & -0.3621849 & -0.0829145 \\ \mathrm{C} & 5.9247479 & -0.0536417 & -0.0825454 \\ \mathrm{C} & 7.7661126 & -1.6698592 & 0.2382433 \\ \mathrm{C} & 4.9838671 & -1.0809058 & 0.2342258 \\ \mathrm{C} & 6.8543704 & -2.6759329 & 0.5522221 \\ \mathrm{C} & 5.4298751 & -2.3810092 & 0.5476639 \\ \mathrm{C} & 7.2893213 & -4.0062446 & 0.8773043 \\ \mathrm{C} & 4.5256151 & -3.4575573 & 0.8573416 \\ \mathrm{C} & 6.3891537 & -4.9948058 & 1.1740271 \\ \mathrm{C} & 4.9860691 & -4.7133885 & 1.1594854 \\ \mathrm{C} & 3.5195790 & -0.7674718 & 0.2332443 \\ \mathrm{C} & 2.8253459 & -0.5408617 & 1.4342194 \\ \mathrm{C} & 2.7944304 & -0.6880535 & -0.9683939 \\ \mathrm{C} & 1.4600000 & -0.2479730 & 1.4339762 \\ \mathrm{C} & 1.4289636 & -0.3957621 & -0.9688254 \\ \mathrm{C} & 0.7301621 & -0.1688679 & 0.2324625 \\ \mathrm{C} & -0.7239799 & 0.1428606 & 0.2327547 \\ \mathrm{C} & -1.5770524 & -0.3517596 & 1.2378135 \\ \mathrm{C} & -1.2999696 & 0.9450191 & -0.7711602 \\ \mathrm{C} & -2.9423060 & -0.0602759 & 1.2377410\end{array}$



C $\quad-2.6637861$
1.2427567
$-0.7675098$
C -3.5148661
0.7448751
0.2361515
C -4.9728509
1.0620664
0.2411995
C -5.4065444
2.4074101
0.5781486
C -5.9214978
0.0484457
$-0.0785284$
C -6.8120137
2.7115416
0.5819977
C -7.3497953
$0.3705423 \quad-0.0548633$
C -7.7491592
1.6781794
0.2657378
$\begin{array}{llll}\text { C } & -5.5501311 & -1.2580894 & -0.4445667\end{array}$
$\begin{array}{llll}\text { C } & -8.2852654 & -0.6332584 & -0.3648725\end{array}$
$\begin{array}{llll}\text { C } & -6.5034422 & -2.2823732 & -0.7618922\end{array}$
$\begin{array}{llll}\text { C } & -7.9029332 & -1.9665447 & -0.7171877\end{array}$
C $-6.1106429-3.5877985-1.1173643$
$\begin{array}{llll}\text { C } & -8.8416327 & -2.9712550 & -1.0265633\end{array}$
$\begin{array}{llll}\text { C } & -7.0604915 & -4.5693763 & -1.4207063\end{array}$
$\begin{array}{llll}\text { C } & -8.4268074 & -4.2608785 & -1.3745669\end{array}$
$\begin{array}{llll}\text { C } & -4.5049140 & 3.4335240 & 0.9281606\end{array}$
$\begin{array}{llll}\text { C } & -7.2371008 & 4.0143074 & 0.9093628\end{array}$
$\begin{array}{llll}C & -4.9516045 & 4.7217752 & 1.2505603\end{array}$
$\begin{array}{llll}\text { C } & -6.3184910 & 5.0162994 & 1.2382690\end{array}$
$\begin{array}{lllll}\mathrm{H} & 9.3435419 & 0.4087166 & -0.3975607\end{array}$
$\begin{array}{llll}\mathrm{H} & 8.8374376 & -1.8972048 & 0.2401973\end{array}$
H $\quad 8.3648746 \quad-4.2122311 \quad 0.8803957$
$\begin{array}{lllll}\mathrm{H} & 6.7360587 & -6.0036770 & 1.4192993\end{array}$
H $4.2751411 \quad-5.5134691 \quad 1.3897609$
H $3.4513001 \quad-3.2606956 \quad 0.8455922$
$\begin{array}{lllll}\mathrm{H} & 4.4707708 & 1.5275188 & -0.3818797\end{array}$
$\begin{array}{llll}\mathrm{H} & 3.3681526 & -0.5851599 & 2.3836451\end{array}$
$\begin{array}{llll}\mathrm{H} & 3.3080895 & -0.8703969 & -1.9175661\end{array}$
H $\quad 0.9573042 \quad-0.0512372 \quad 2.3856812$
H $\quad 0.8902344 \quad-0.3697578 \quad-1.9208593$
$\begin{array}{llll}\mathrm{H} & -1.1704482 & -0.9959548 & 2.0232415\end{array}$
$\begin{array}{lllll}\mathrm{H} & -0.6658008 & 1.3643811 & -1.5580890\end{array}$
$\begin{array}{llll}\mathrm{H} & -3.5804973 & -0.4647396 & 2.0295005\end{array}$
$\begin{array}{llll}\mathrm{H} & -3.0795991 & 1.8754681 & -1.5578276\end{array}$
$\mathrm{H} \quad-5.0430466 \quad-3.8292362 \quad-1.1538994$
$\begin{array}{lllll}\mathrm{H} & -6.7341228 & -5.5776623 & -1.6943453\end{array}$
H $\quad-9.1711264-5.0276042 \quad-1.6117321$
$\begin{array}{llll}\mathrm{H} & -9.9092742 & -2.7294462 & -0.9928074\end{array}$
$\begin{array}{lllll}\mathrm{H} & -4.4902622 & -1.5167941 & -0.4985827\end{array}$
$\mathrm{H} \quad-9.3512459-0.3834520 \quad-0.3403303$
$\begin{array}{llll}\mathrm{H} & -8.8171676 & 1.9192521 & 0.2760474\end{array}$
$\begin{array}{llll}\mathrm{H} & -8.3099630 & 4.2340922 & 0.9067193\end{array}$
$\begin{array}{llll}\mathrm{H} & -6.6707363 & 6.0217024 & 1.4894300\end{array}$
$\begin{array}{llll}\mathrm{H} & -4.2234833 & 5.4948423 & 1.5155962\end{array}$
H $\quad-3.4341630 \quad 3.2195692 \quad 0.9532100$ 


$\begin{array}{cccc}\text { 1,3-Bis(tetracen-5-yl)benzene } & (\mathbf{3}) \\ \text { C } & -3.6988701 & -0.4873687 & 6.1989711 \\ \text { H } & -4.4459851 & -0.4121350 & 6.9953866 \\ \text { C } & -2.4891620 & 0.2057469 & 6.3108370 \\ \text { C } & -1.5110841 & 0.1252465 & 5.2992251 \\ \text { C } & -1.7706709 & -0.6823718 & 4.1413998 \\ \text { C } & -2.9970059 & -1.3700406 & 4.0515647 \\ \text { C } & -3.9533058 & -1.2758086 & 5.0685908 \\ \text { H } & -2.2910647 & 0.8231902 & 7.1934245 \\ \text { C } & -0.2634985 & 0.8223829 & 5.3723372 \\ \text { C } & -0.7730364 & -0.7625745 & 3.1132684 \\ \text { H } & -3.1958466 & -1.9871935 & 3.1688628 \\ \text { H } & -4.8995148 & -1.8187502 & 4.9793976 \\ \text { C } & 0.7147039 & 0.7411612 & 4.3646406 \\ \text { C } & 0.4625300 & -0.0942649 & 3.1886221 \\ \text { C } & 1.9237649 & 1.4500967 & 4.4513647 \\ \text { C } & 1.4534838 & -0.1794107 & 2.1682463 \\ \text { C } & 2.9292376 & 1.3805539 & 3.4364146 \\ \text { C } & 2.7024278 & 0.5549014 & 2.2810759 \\ \text { C } & 4.1390982 & 2.0933101 & 3.5511931 \\ \text { C } & 3.7232381 & 0.4775254 & 1.3114305 \\ \text { C } & 5.1280716 & 2.0078712 & 2.5660473 \\ \text { C } & 4.9186611 & 1.1954536 & 1.4474720 \\ \text { C } & 1.2105940 & -1.0320282 & 0.9667712 \\ \text { C } & 0.9786568 & -0.4486138 & -0.2924504 \\ \text { C } & 1.2067741 & -2.4354271 & 1.0648564 \\ \text { C } & 0.7447544 & -1.2292422 & -1.4369954 \\ \text { C } & 0.9763115 & -3.2248890 & -0.0652403 \\ \text { C } & 0.7449981 & -2.6288861 & -1.3086474 \\ \text { C } & 0.5033320 & -0.5804552 & -2.7661176 \\ \text { C } & 1.5473305 & -0.4939425 & -3.7092832 \\ \text { C } & -0.7883464 & -0.0526567 & -3.0708500 \\ \text { C } & 1.3068040 & 0.1332874 & -4.9995372 \\ \text { C } & -1.0165724 & 0.5757861 & -4.3630556 \\ \text { C } & 0.0443581 & 0.6472373 & -5.2895968 \\ \text { C } & 2.8711972 & -0.9965597 & -3.4496886 \\ \text { C } & 2.3836832 & 0.2119281 & -5.9475380 \\ \text { C } & 3.8698245 & -0.8980641 & -4.3843795 \\ \text { C } & 3.6251723 & -0.2869041 & -5.6550737 \\ \text { C } & -1.8741513 & -0.1223535 & -2.1654584 \\ \text { C } & -3.355514991 & 1.0967609 & -4.6637968 \\ & 0.3955086 & -2.4709174 \\ & 1.0266500 & -3.7597264\end{array}$




$\begin{array}{rrrr}\mathrm{C} & -4.2330505 & 0.3254536 & -1.5470699 \\ \mathrm{C} & -4.6594211 & 1.5524990 & -4.0544102 \\ \mathrm{C} & -5.4618792 & 0.8405879 & -1.8693644 \\ \mathrm{C} & -5.6783001 & 1.4630373 & -3.1418999 \\ \mathrm{H} & -0.0640856 & 1.4482059 & 6.2485831 \\ \mathrm{H} & 2.1078168 & 2.0797224 & 5.3279933 \\ \mathrm{H} & 4.2984789 & 2.7206542 & 4.4345355 \\ \mathrm{H} & 6.0603790 & 2.5707743 & 2.6751203 \\ \mathrm{H} & 5.6879798 & 1.1142958 & 0.6732228 \\ \mathrm{H} & 3.5813946 & -0.1577443 & 0.4344264 \\ \mathrm{H} & -0.9974795 & -1.3727246 & 2.2355479 \\ \mathrm{H} & 4.8674877 & -1.2886154 & -4.1591389 \\ \mathrm{H} & 3.0748277 & -1.4650147 & -2.4840039 \\ \mathrm{H} & 4.4356161 & -0.2189510 & -6.3877620 \\ \mathrm{H} & 2.1866846 & 0.6847343 & -6.9154621 \\ \mathrm{H} & -0.1319931 & 1.1198847 & -6.2616466 \\ \mathrm{H} & -2.4540187 & 1.5682290 & -5.6397063 \\ \mathrm{H} & -4.8229497 & 2.0283078 & -5.0271182 \\ \mathrm{H} & -6.6670375 & 1.8684350 & -3.3797111 \\ \mathrm{H} & -6.2882342 & 0.7808010 & -1.1538743 \\ \mathrm{H} & -4.0659397 & -0.1481999 & -0.5740232 \\ \mathrm{H} & -1.7254380 & -0.5965548 & -1.1923859 \\ \mathrm{H} & 0.5632952 & -3.2501939 & -2.1910138 \\ \mathrm{H} & 0.9782280 & -4.3162793 & 0.0237580 \\ \mathrm{H} & 1.3909244 & -2.9067643 & 2.0350651 \\ \mathrm{H} & 0.9780904 & 0.6418472 & -0.3830154\end{array}$

\section{References:}

1. Ahlrichs, R.; Bär, M.; Häser, M.; Horn, H.; Kölmel, C. Chem. Phys. Lett. 1989, $162,165-169$.

2. Schäfer, A.; Horn, H.; Ahlrichs, R. J. Chem. Phys. 1992, 97, 2571-2577.

3. Bauernschmitt, R.; Ahlrichs, R. Chem. Phys. Lett. 1996, 256, 454-464. 